\title{
Review
}

\section{DNA repair gene status in oesophageal cancer}

\author{
R Naidoo, R Chetty
}

\begin{abstract}
DNA repair genes and microsatellite instability (MSI) are relatively recently described molecular events that have been associated particularly with colorectal cancers in the setting of hereditary non-polyposis colorectal cancer or the Lynch syndromes. Several other gastrointestinal (and other) malignancies have been analysed for abnormalities in DNA repair genes and MSI. Dietary and environmental factors have been implicated strongly in the aetiology of oesophageal cancer. However, the effect of this on the genetic profile, especially the DNA repair system and resultant MSI, is largely unknown. The purpose of this review is to provide a brief background of the dietary and environmental factors in oesophageal carcinogenesis and to discuss the role of the repair genes and MSI in the molecular pathogenesis of this malignancy. Several studies indicate that MSI (range, 3-40\%) and loss of heterozygosity (LOH) (range, 3-64\%) in the DNA repair genes are uncommon in carcinogenesis of the oesophagus. Most data are at the lower end of the ranges and this, together with the lack of uniform criteria for the assessment of MSI, accounts for the higher figures obtained in some studies. The rates of detection of MSI do not approach that of other gastrointestinal malignancies, such as gastric (up to $23 \%$ ) and colorectal (up to $31 \%$ ) carcinomas.
\end{abstract}

(F Clin Pathol: Mol Pathol 1999;52:125-130)

Keywords: oesophageal cancer; microsatellite instability; DNA repair genes

Molecular Biology Research Facility and the Department of Pathology, University of Natal School of Medicine, Congella 4013, Durban, South Africa

R Naidoo

R Chetty

Correspondence to: Professor Chetty.

email:

chettyr@med.und.ac.za

Accepted for publication 21 January 1999 There are several factors that influence the pathogenesis of oesophageal carcinoma. Certain countries have a higher incidence of the disease and these include China, Japan, Switzerland, France, Finland, certain parts of Africa (especially Southern Africa), and Iran. There are also local variations within single countries.

There are a number of predisposing factors that seem to influence the pathogenesis of oesophageal carcinoma. However, in most reports there is no clear separation of speculative and proven aetiological factors. Poor oral hygiene, mechanical trauma, dietary intake, physiological narrowing of the oesophagus, syphilis, and megaoesophagus have all been proposed (among others) as predisposing factors, without convincing proof. In particular, adenocarcinoma of the oesophagus is associated with Barrett's oesophagus: patients with this disease have a 30-40 times greater risk of developing adenocarcinoma compared with the general population.

In this review we will present dietary and environmental risk factors, before dwelling on the putative role of the DNA repair genes and microsatellite instability (MSI) in oesophageal carcinogenesis.

\section{Dietary and environmental factors}

Scientists now believe that one third of all cancers are related to what we eat. High salt diets containing large amounts of smoked and salt pickled foods are said to be high risk factors for oesophageal carcinoma. Nitrates and nitrosamines, which are found in many preserved foods, are thought to be potent carcinogens. Increased intake of foods containing these compounds promotes the development of oesophageal tumours. In addition, alcohol ingestion has also been implicated in the aetiology of oesophageal cancer.

Another factor that has been linked to the development of oesophageal cancer is tobacco smoking. Tobacco contains more than 4000 chemical compounds, of which 43 are confirmed carcinogens (published by the American Association for Cancer Research). The higher incidence of oesophageal carcinoma among those who smoke or chew tobacco implicates nicotine and other carcinogenic agents found in tobacco in the aetiology of this disease.

Oesophageal cancer is the most common 
in the development of oesophageal cancer. A vastly increased incidence of the disease in the Transkei region might be related to a deficiency of molybdenum or a disturbance of the zinc/copper ratio in the soil. Furthermore, epidemiological studies suggest that $\sim 80-90 \%$ of cancers are attributable to lifestyle, with $50 \%$ being diet related. Various genotypes of human papilloma virus have also been detected in oesophageal cancers; however, their specific role is not clear.

Allied to these environmental risk factors is the influence of molecular genetic alterations that might accrue from the aforementioned factors or precede these environmental influences. Several genes have been implicated in the aetiology of oesophageal carcinoma: p53, the retinoblastoma gene, APC (adenomatous polyposis coli), MCC (mutated in colorectal cancer), and DCC (deleted in colorectal cancer). In oesophageal carcinomas, p53 gene abnormalities are some of the most common aberrations and occur in $\sim 33-50 \%$ of cases. Hollstein and colleagues were some of the first to show the role of specific p53 mutations in the tumorigenic process in oesophageal cancer. ${ }^{4}$ The patients examined in their study were from high risk geographical areas. Mutations of the p53 gene have been described in both squamous carcinomas and adenocarcinomas of the oesophagus, as well as their respective precursor lesions.

\section{DNA damage}

DNA damage with resultant mismatched base pairs is generated by:

- Damage to nucleotide precursors.

- Errors that occur during replication.

- Genetic recombination.

- Double strand DNA breaks that lead to chromosome disruption. ${ }^{5}$

Damage resulting from any of the above mechanisms results in pronounced cellular dysfunction. The errors arise as a result of environmental insults that damage specific nucleotides. An example of this is UV damage to DNA. Here, UV light can lead to crosslinking of pyrimidine (thymidine or cytosine) residues. The dimers that form as a result block normal DNA replication. Another type of injury to the DNA results from the covalent addition of chemical groups to the nucleotides (alkylation). In addition, purines (adenine or guanine) spontaneously break off from the DNA backbone because of intrinsic thermal decompression. Finally, spontaneous deaminations occur at cytosine residues, resulting in the formation of uracil residues. Because uracil is not one of the bases usually found in DNA, transcription is blocked when DNA polymerase encounters one of these altered residues. DNA damage as a result of these errors is corrected by the process of nucleotide excision repair. ${ }^{6}$

\section{Mechanisms of DNA repair}

Excision repair is classified into two distinct types, known as "base excision repair" and "nucleotide excision repair". During base excision repair, damaged bases are excised as free bases whereas, in nucleotide excision repair, the offending base is excised as a free nucleotide. Nucleotide excision repair, sometimes referred to as "short patch repair", is said to operate efficiently in DNA that has been damaged by chemical modification, such as the production of cyclobutane pyrimidine dimers, which causes distortion of the helix. Mismatch repair has been investigated intensively in Escherichia coli and the yeast Saccharomyces cerevisiae. The relation between defects in mismatch repair and human disease has been examined closely, with particular interest in the development of cancer.

The mechanism of action in nucleotide excision repair is outlined in fig 1 . There are two proteins, UvrA and UvrB, that bind to structurally damaged DNA in an ATP dependent manner. The UvrC protein then binds and cleaves the damaged strand at two sites, 12 base pairs apart, flanking the damaged nucleotides. Helicase II unwinds the DNA and then polymerase I excises the nucleotides between the two nicks and resynthesises the strand. The final step in the process is closure of the gap, which is accomplished by DNA ligase.

The second way in which DNA can be altered is by mismatching of nucleotides. DNA duplication occurs with every mitotic division and errors could be introduced during duplication; therefore, an extremely precise proofreading mechanism must exist. Although very precise, DNA polymerase is not $100 \%$ accurate.

In the $E$ coli system, the mismatch repair pathway recognises all single base mismatches, except possibly C-C. In addition, all small insertion mismatches are repaired; however, sections that have more than four mispaired bases are not recognised efficiently by this system. The system recognises the mispaired bases that arise as a result of misincorporation during DNA synthesis. Furthermore, cells that lack this repair system pathway have a high rate of accumulation of mutations.

The MutHLS repair pathway is unique in that it is suited to correct DNA replication errors. In the $E$ coli system, GATC sites are usually methylated and this is achieved by the enzyme Dam methylase. However, after replication the daughter strand is transiently unmethylated. The repair system recognises these unmethylated sites on the daughter strand and selectively corrects this.

\section{DNA repair genes}

Recently, a new class of genes, known as the DNA mismatch repair genes, has been identified. These genes encode proteins that play the role of "proofreaders" by ensuring that the copies of new DNA produced in the cell have the same genetic make up or sequence as the parent strands. The repair of biosynthetic errors, such as alterations in microsatellite length and single base mismatches, is a highly conserved cellular function, which is carried out by proteins that recognise these defective sequences and excise and replace them with the correct ones. Enzyme systems that faithfully repair these aberrations have been identified in a wide variety of organisms. ${ }^{8}$ However, 


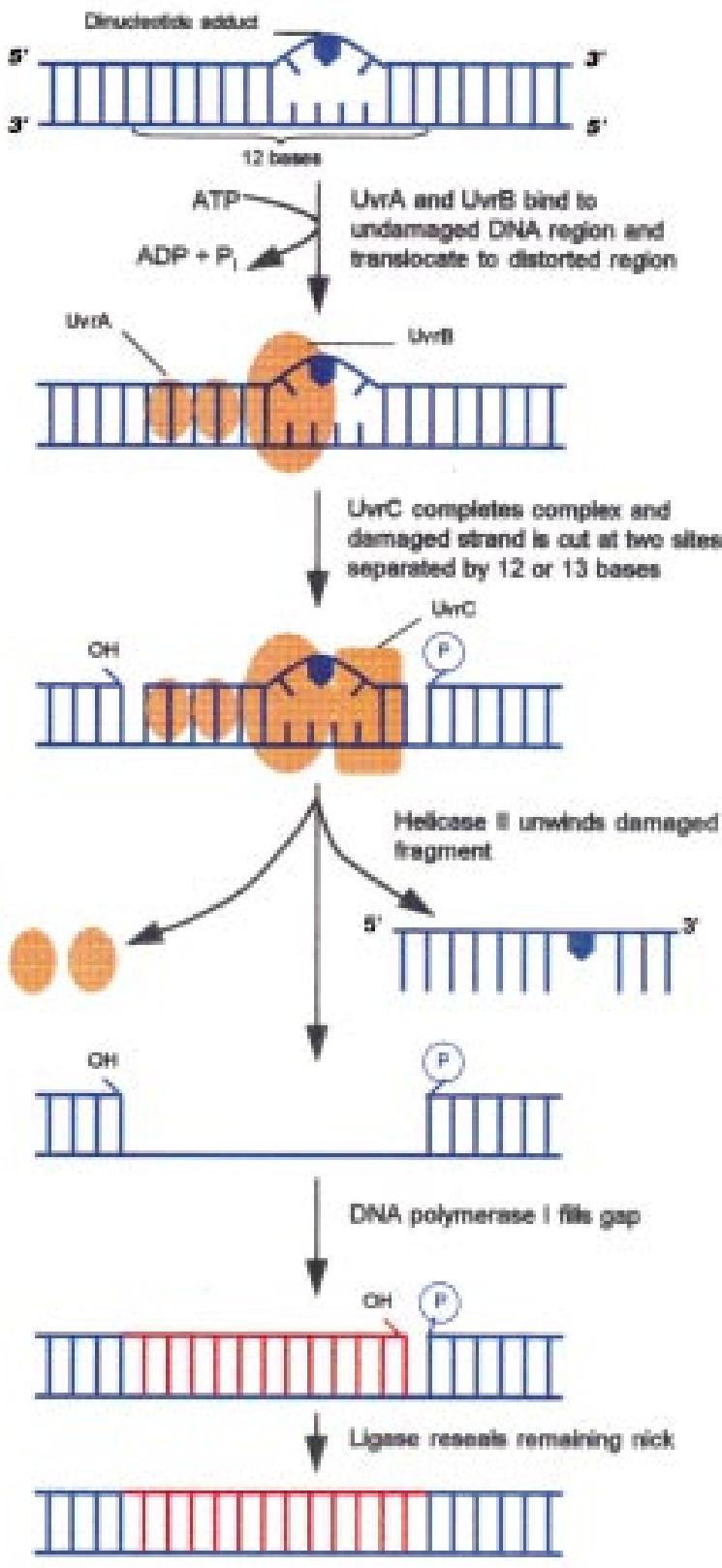

Figure 1 A diagrammatic representation of the process of nucleotide excision repair. The damaged segment of DNA is cut out and the defect or gap is then repaired to re-establish the normal sequence. agents/events, such as chemicals or toxins. Defects in these repair genes lead to genetic instability and they are therefore thought to play a pivotal role in oncogenesis.

The two most extensively studied repair genes are $\mathrm{hMSH} 2$, which is located on chromosome $2 \mathrm{p}$, and hMLH1, located on chromosome 3p. Essentially, both hMSH2 and hMLH1 act as tumour suppressor genes, with loss of both copies of the gene resulting in abnormalities in the mismatch repair system.

The proteins encoded by the DNA repair genes are thought to act as a complex and mutations in any of these genes can give rise to MSI. It is envisaged that these alterations act as triggers for the initiation and pathogenesis of many tumours.

The normal functions of the proteins encoded by these genes are outlined in fig 2 . The correction of a replication error is initiated by the binding of the $97 \mathrm{kDa}$ MutS protein, which recognises and binds to the mismatched DNA sequence. The DNA exists as a heteroduplex because of the lack of complementarity of the bases. The MutL (a $70 \mathrm{kDa}$ dimer) and the MutH (a $25 \mathrm{kDa}$ monomer) proteins then act together with the already bound MutS protein. MutH induces a nick in the strand containing the incorrect nucleotide. The enzyme helicase II then unwinds the DNA strand on both sides of the nick. This is followed by the bidirectional removal of bases between the nick and the mismatched base. The gap is then filled with the correct nucleotide sequence by DNA polymerase and the newly synthesised strand is finally sealed by DNA ligase. Figure 3 reflects the situation when effective DNA repair is not accomplished and MSI or DNA slippage results.

Less is known about the homologues hPMS1, hPMS2, and GTBP. ${ }^{8}$ The products of these genes are also thought to act as a complex and mutations in any of these genes also give rise to MSI. Mismatch repair has long been known to play two major roles in the cell, namely: as a repair mechanism for errors arising during DNA synthesis and in genetic recombinations that result in new genetic markers. It is envisaged that these genetic alterations act as a trigger for the initiation and pathogenesis of many tumours.

mutations in the genes encoding these proteins result in defective proteins that fail to correct replication errors. This has a cascade effect, producing secondary mutations further downstream from the initial mutation site. Sometimes these secondary mutations occur in oncogenes and tumour suppressor genes. At present, six genes have been identified that encode mismatch repair proteins.

These genes were identified originally in bacteria and yeast, ${ }^{9}$ and their human homologues have been implicated in the pathogenesis of hereditary non-polyposis colorectal cancer, as well as a host of different sporadic cancers that exhibit MSI. The normal DNA mismatch repair genes maintain the integrity of the DNA by repairing errors that occur during DNA replication or after exposure to genotoxic

\section{Microsatellite instability}

Alterations resulting from mutations in the simple repeat sequences or microsatellites are a feature of many tumours. ${ }^{10-12}$ It is envisaged that the analysis of MSI may be important for cancers. ${ }^{13}$ The eukaryotic genome contains not only introns and exons, but also large numbers of copies of other seemingly non-essential encode proteins and in many regions are "repetitious". The variation within these repetitive stretches of DNA is so great that each human can be distinguished by a "DNA fingerprint". This fingerprint is based on the variation in the repetitive sequences. identifying a substantial fraction of human DNA (>90\%). This is occasionally referred to as "junk DNA". These DNA sequences do not 

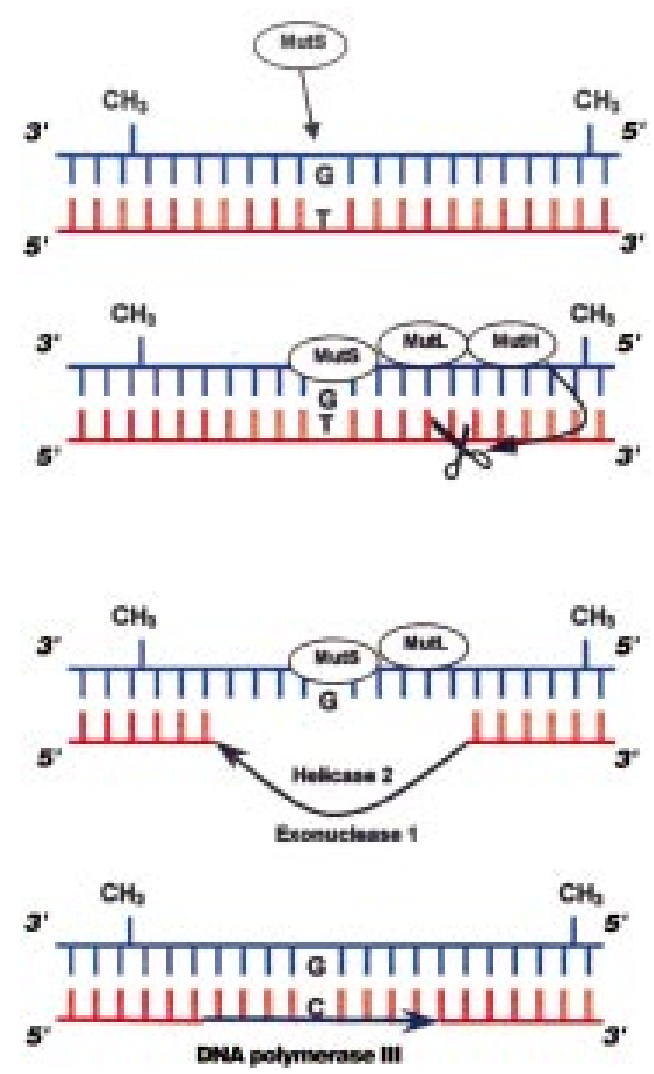

Figure 2 This diagram of an Escherichia coli strain shows how the DNA repair genes usually work. MutS and MutL are the homologues of the human genes hMSH and hMLH1, respectively. The MutH homologue in humans has not yet been identified. Once the site of the mismatched nucleotides is detected, the repair genes bind to this site. The MutH protein induces a nick in the DNA molecule near the mismatch site. The abnormal area is then excised with the aid of DNA helicase 2 and an exonuclease 1. The resultant gap in the DNA is then filled by the DNA polymerase III enzyme.

About a third of all these repeat sequences are short tandem repeat sequences referred to as microsatellites, which are scattered throughout the human genome. The lengths of these microsatellites are unique to each individual and vary between individuals; however, there are no variations between different cells in the same individual. ${ }^{14}$ In addition, these sequences are highly conserved and are stably inherited. ${ }^{15}$

Microsatellites are located in the heterochromatin near chromosomal centromeres and telomeres and between 55000 and 100000 copies are estimated to occur in the human genome, providing a marker density of one microsatellite every 100000 bp. ${ }^{16}$ However, although widely distributed, microsatellites are not regularly spaced in the chromosome.

Weissenbach et al. isolated and mapped a large number of microsatellites to construct a linkage map of the human genome with an average resolution of 5 centimorgans $(\mathrm{cM}) .{ }^{17}$ The sequence data obtained from this study are widely used for various microsatellite applications. Microsatellites can be used for personal identification, population genetic analysis, and in the construction of the human evolutionary tree. ${ }^{18}$ Furthermore, they are located at and linked to several important gene loci; thus, they are associated with human diseases not only as markers but also directly in disease aetiopathogenesis, providing insight into the replication, repair, and mutation of eukaryotic DNA. ${ }^{19}$ Microsatellites were thought initially to play a functional role in the genome, either directly in gene regulation, or indirectly as hot spots for recombination; however, their exact function still remains elusive. Recent studies have shown that mutations occur in these microsatellites as a result of mismatch repair or replicative errors (RERs). These mutations are caused by an increase or decrease in the number of repeats. As a result, different lengths of DNA are produced that arise directly from the defective repair process. The mutations that occur are referred to as microsatellite instability, which has been implicated in a host of human disorders, both hereditary and non-hereditary, including colorectal, breast, and prostatic cancers.

There has not been a uniform definition of either MSI or RER. A recent review has used the following criteria: the presence of microsatellite instability in at least one locus is termed MSI positive, while it is proposed that for a case to be RER positive, it should display MSI in at least $29 \%$ of the microsatellite loci examined. ${ }^{20}$

\section{MSI in the DNA repair genes in} oesophageal carcinoma

MSI has not been investigated extensively in oesophageal cancer; most studies have been in squamous carcinomas.

OESOPHAGEAL ADENOCARCINOMA

A study undertaken recently to determine MSI in oesophageal cancer showed that it occurred more frequently in adenocarcinomas than in squamous carcinomas. ${ }^{21}$ The 106 patients studied included 28 patients with Barrett's metaplasia, 36 with Barrett's associated adenocarcinoma, and 42 with primary oesophageal squamous cell carcinoma. MSI was noted in nine (one squamous cell carcinoma and eight adenocarcinomas) of the 106 patients $(8.5 \%)$, in the $2 \mathrm{p}$ region. In Barrett's metaplasia alone, MSI was not found in the $2 \mathrm{p}$ region. This study suggests that MSI is common in oesophageal adenocarcinoma. However, with the varying application of definitions of MSI, this figure is lower than reported originally. Muzeau and colleagues examined 20 adenocarcinomas derived from Barrett's metaplasia for MSI in the $3 p$ region, but did not give specific details of the results for each marker. ${ }^{22}$ However, this particular study is the only one that has examined a substantial number of patients. A study by Wang et al examined two adenocarcinomas of the oesophagus, but did not separate these results from those obtained with squamous carcinomas. ${ }^{23}$ Thus, well defined information of the status of the DNA repair genes in oesophageal adenocarcinoma is scanty and difficult to interpret.

OESOPHAGEAL SQUAMOUS CARCINOMA

Analysis of the $2 p$ and $3 p$ loci in another study also showed that a very low percentage $(3 \%$ and $9 \%$, respectively) of cases demonstrated MSI. ${ }^{24}$ Although no significant correlation was 
shown between MSI analysis and clinicopathological findings, some of their cases correlated with DNA replication error. Alternatively, Ogasawara et al detected MSI and loss of heterozygosity $(\mathrm{LOH})$ in a high percentage of their cases (up to $60 \%$ MSI). ${ }^{25}$ Their results suggest that RER at the $3 p$ locus is an early event and that a tumour suppressor gene involved in the progression of oesophageal cancer might exist in the vicinity of the 3 p25 locus.

A recent study from a Chinese group using a total of 12 microsatellite markers found MSI and $\mathrm{LOH}$ at the $3 \mathrm{p}$ locus to be $66.7 \%$ and $42 \%$, respectively. ${ }^{23}$ Although these last two studies have demonstrated a high degree of MSI in oesophageal cancers, a recent finding by Muzeau et al failed to detect MSI or LOH at the $2 p$ and $3 p$ regions in their cohort of 20 squamous cell carcinomas. ${ }^{22}$ Their study analysed 39 poly-CA microsatellite loci in the French population. ${ }^{22}$

In the study by Mori and colleagues, 21 squamous cancers were analysed with markers to the 3 p region. ${ }^{26}$ They found that nine of 14 informative cases exhibited LOH. It must be borne in mind that in the preceding studies, radioactively labelled probes and not fluorescence based technology were used.

We conducted a microsatellite analysis using fluorescently labelled primers on 39 oesophagectomy specimens for squamous car-
Table 1 Microsatellite instability (MSI) and loss of heterozygosity (LOH) for the DNA repair genes in squamous cell oesophageal carcinoma

\begin{tabular}{|c|c|c|c|}
\hline Authors & $\begin{array}{l}\text { Case } \\
\text { numbers }\end{array}$ & Locus $(2 p)$ & Locus (3p) \\
\hline Meltzer et al ${ }^{11}$ & 42 & MSI $2 \%$ & B \\
\hline Muzeau et a $l^{2}$ & 46 & LOH $0 \%$ & LOH $0 \%$ \\
\hline Wang et $a l^{23}$ & 34 & $\begin{array}{l}\text { MSI } 0 \% \\
-\end{array}$ & $\begin{array}{l}\text { MSI 0\% } \\
\text { LOH } \\
12-30 \% \text { MSI } \\
6-30 \%\end{array}$ \\
\hline Nakashima et al ${ }^{4}$ & 32 & $\begin{array}{l}\text { LOH 3\% } \\
\text { MSI 3\% }\end{array}$ & $\begin{array}{l}\text { LOH } 0 \% \\
\text { MSI } 9 \%\end{array}$ \\
\hline Ogasawara et al ${ }^{5}$ & 35 & $\begin{array}{l}\text { LOH 3\% } \\
\text { MSI } 20 \%\end{array}$ & $\begin{array}{l}\text { LOH } 3-35 \% \\
\text { MSI } 13-40 \%\end{array}$ \\
\hline Mori et $a l^{6}$ & 21 & - & LOH $64 \%$ \\
\hline Aoki $e t a l^{77}$ & 93 & - & LOH 35\% \\
\hline Wagata $e t a l^{28}$ & 35 & - & LOH $10 \%$ \\
\hline Shibagaki et al ${ }^{9}$ & 36 & - & LOH $41 \%$ \\
\hline $\begin{array}{l}\text { Naidoo et al (unpublished } \\
\text { data, 1998) }\end{array}$ & 39 & $\begin{array}{l}\text { LOH } 30 \% \\
\text { MSI } 10 \%\end{array}$ & $\begin{array}{l}\text { LOH } 24-33 \% \\
\text { MSI } 8 \%\end{array}$ \\
\hline
\end{tabular}

cinoma with three markers for the $2 p$ and $3 p$ regions (unpublished data, 1998). MSI was not found in the D3S659 locus, whereas for $\mathrm{D} 3 \mathrm{~S} 1255$ and $\mathrm{D} 2 \mathrm{~S} 123$, it was $8.3 \%$ and $10.25 \%$, respectively. For the D2S123 marker, $\mathrm{LOH}$ or allelic imbalance was found in $30 \%$ of cases, while in the $3 \mathrm{p}$ region, $\mathrm{LOH}$ ranged from $24 \%$ (D3S1255) to 33\% (D3S659). Table 1 summarises the results of the various studies into MSI and LOH of the DNA repair genes in squamous cell oesophageal carcinoma.

Thus, the phenomenon of MSI appears to be infrequent in oesophageal cancer, and this is
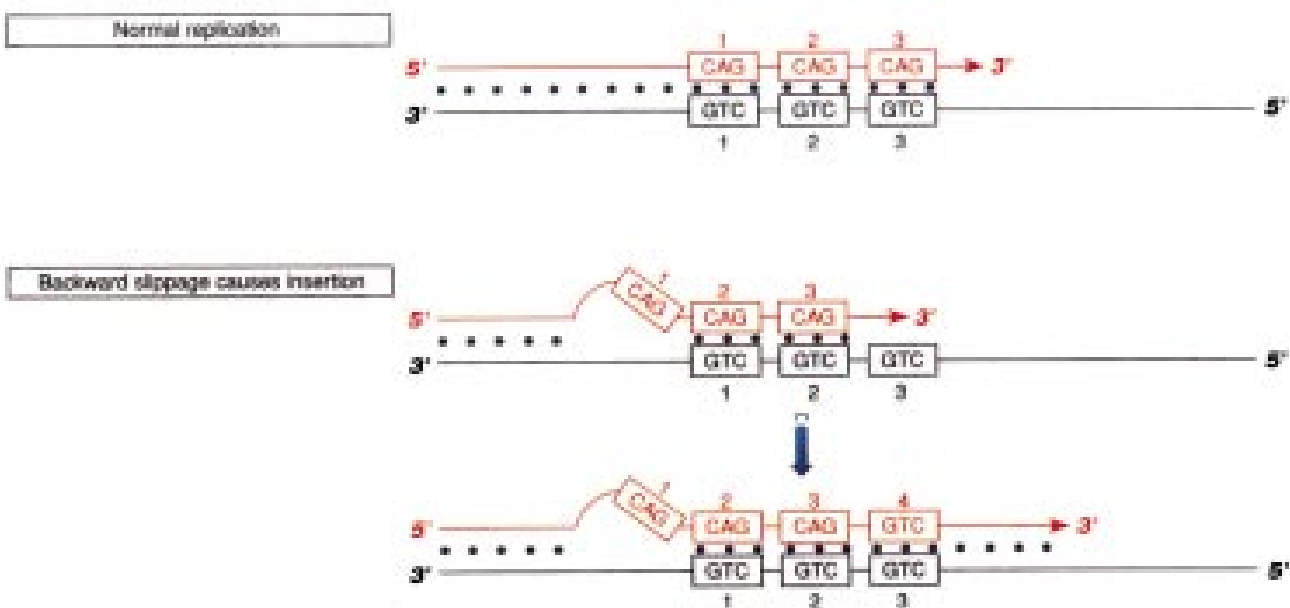

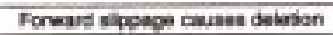

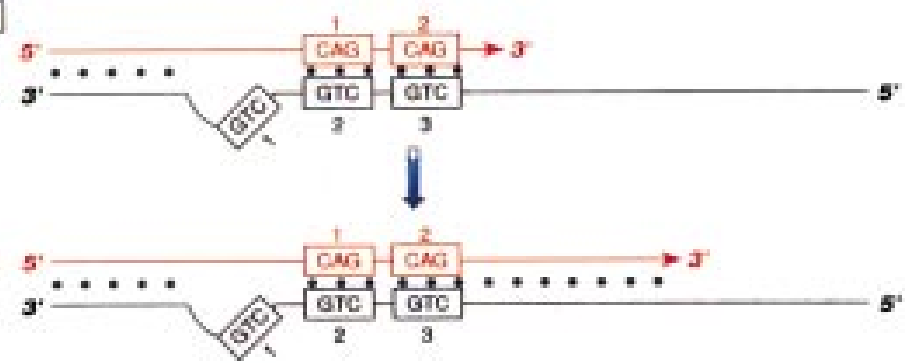

Figure 3 These are examples of how the phenomenon of microsatellite instability arises. The upper strand (red) represents the newly synthesised complemetary strand of DNA, while the lower strand (black) is the parent strand. During backward slippage there is a region of non-pairing (CAG 1, shown as a bubble on the upper strand) containing one or more repeats of the newly synthesised strand. This results in an increase in the repeat units or insertions. During forward slippage, there is a region of non-pairing (GTC 1, shown as a bubble on the lower strand), which results in a decrease in the number of repeat units or deletions in the newly synthesised strand. 
the experience of several workers from different geographical areas. Although there are two notable exceptions, ${ }^{23}{ }^{25}$ this might be the result of technical differences. The use of fluorescence based primers together with computer assisted software gives an objective interpretation of the bands. The problem of interpretation of "stutter bands" is a well recognised pitfall when assessing microsatellite data.

Therefore, abnormalities in the DNA repair genes seem to have a limited role in the molecular pathogenesis of oesophageal cancer.

$\mathrm{RN}$ and RC are supported by the Medical Research Council of South Africa, Kennedy Potts Cancer Endowment, and the Cancer Association of South Africa.

1 Morson B, Dawson I. Gastrointestinal pathology. London: Blackwell Scientific Publications, 1979:33-57.

2 Haffejee A. Surgical research-reflections and a vision. $S A f r$ F Surg 1998;36:10-16.

3 Dayne N, Munoz N. Esophagus. In: Schottenfeld D, Fraumeni J, eds. Cancer epidemiology and prevention. meni J, eds. Cancer epidemiology and 4 Hollstein M, Metcalf R, Welsh J, et al. Frequent mutation of
the p53 gene in human esophageal cancer. Proc Natl Acad the p53 gene in human esoph

$5 \mathrm{Chu}$ G. Double strand break repair. If Biol Chem 1997;26:24097-100.

6 Wood R. DNA repair in eukaryotes. Annu Rev Biochem 1996;65:135-67.

7 Kolodner R. Mismatch repair: mechanisms and relationship to cancer suceptibility. Trends Biochem Sci 1995;20:397401.

8 Fishel R, Kolodner R. Identification of mismatch repair genes and their role in the development of cancer. Curr Opin Genet Dev 1995;5:382-95.

9 Modrich P, Laheu R. Mismatch repair in replication fidelity, genetic recombination, and cancer biology. Annu Rev Biochem 1996;65:101-33.

10 Todd J. La carte des microsatellites est arrivee! Hum Mol Genet 1992;1:663-6.

11 Horii A, Han H, Shimada M, et al. Frequent replication errors at microsatellite loci in tumors of patients with multiple primary cancers. Cancer Res 1994;54:3373-5.
12 Brentnall T. Microsatellite instability - shifting concepts in tumorigenesis. Am f Pathol 1995;147:561-3.

13 McCarthy K. Microsatellites: heroes and villains. Lancet 1995;346:1177.

14 Beckman S, Weber J. Survey of human and rat microsatellites. Genomics 1992;12:627-31.

15 Weber J, May P. Abundant class of human DNA polymorphisms which can be typed using polymerase chain reaction. Am f Hum Genet 1989;44:388-96.

16 Koreth J, O'Leary J, McGee J. Microsatellite and PCR genomic analysis. F Pathol 1996;178:239-48.

17 Weissenbach J, Gyapay G, Dib C, et al. A second generation linkage map of the human genome. Nature 1992;359:794 801.

18 Di Rienzo A, Peterson A, Garza J, et al. Mutational processes of simple-repeat loci in human populations. Proc Natl Acad Sci USA 1994;91:3166-70.

19 Meltzer S. Dr Strange DNA, or how I learned to stop cloning and love the computer. Gastroenterology 1995;109:611-

20 Hayden J, Martin I, Cawkwell L, et al. The role of microsatellite instability in gastric carcinoma. Gut 1998;42:300-3.

21 Meltzer S, Yin J, Manin B, et al. Microsatellite instability occurs frequently and in both diploid and aneuploid cell populations of Barrett's-associated esophageal adenocarcinoma. Cancer Res 1994;54:3397-82.

22 Muzeau F, Flejou J, Belghiti J, et al. Infrequent microsatellite instability in oesophageal cancers. Br F Cancer 1997;75: 1336-9.

23 Wang L, Li W, Wang X, et al. Genetic alterations on chromosome 6 and 9 of esophageal cancer tissue from China. Oncogene 1996;13:699-703.

24 Nakashima H, Mori M, Mimori K, et al. Microsatellite instability in Japanese esophageal carcinoma. Int $\mathcal{f}$ Cancer 1995;64:286-9.

25 Ogasawara S, Maesawa C, Tamura G, et al. Frequent microsatellite alterations on chromosome $3 p$ in esophageal squamous cell carcinoma. Cancer Res 1995;55:891-4.

26 Mori T, Yanaglsawa A, Kato Y, et al. Accumulation of genetic alterations during esophageal carcinogenesis. Hum Mol Genet 1994;3:1969-71.

27 Aoki T, Mori T, Du X, et al. Allelotype study of esophageal carcinoma. Genes Chromosomes Cancer 1994;10:177-82.

28 Wagata $T$, Ishizaki $\mathrm{K}$, Imamura $\mathrm{M}$, et al. Deletion of $17 \mathrm{p}$ and amplification of int-2 gene in esophageal carcinomas. Cancer Res 1991;51:2113-17.

29 Shibagaki I, Shimada Y, Wagata T, et al. Alleotype analysis of esophageal squamous cell carcinoma. Cancer Res 1994;54: 2996-3000. 